\title{
Sex differences in spatial search and pattern learning in the rat
}

\author{
BRIAN K. LEBOWITZ and MICHAEL F. BROWN \\ Villanova University, Villanova, Pennsylvania
}

\begin{abstract}
Male and female rats searched in a $5 \times 5$ matrix of 15.5-cm-tall wooden poles for reward pellets hidden on top of the poles. The baited poles always formed a square pattern, but were otherwise unpredictable. Measures developed by Brown and Terrinoni (1996) were used to determine choice efficiency and the extent to which choices were determined by the spatial pattern. Male rats were controlled by the spatial pattern in which poles were baited to a greater extent than female rats, whereas female rats were more efficient at searching for baited poles when no information about their location was available.
\end{abstract}

The ability to process, store, and utilize spatial information from the environment is integral to the daily functioning of an organism. A growing body of evidence suggests that both human males (Halpern, 1992) and a variety of male animals (Beatty, 1984, 1992) perform more accurately than females on some tasks that are thought to involve spatial ability. Typically, mazes have been used to compare the ways in which male and female laboratory rats use spatial information to navigate through the environment. Mazes such as the Lashley III, the HebbWilliams, the Morris water maze, and the radial arm maze (RAM) have all been used. In general, adult male rats have been found to make fewer errors, and to learn complex mazes faster than females (see Beatty, 1979, 1992, and Williams \& Meck, 1991, for reviews).

Recently, the apparatus most commonly used to examine sex differences in spatial ability in the rat has been the RAM. Some RAM studies have shown a sex difference favoring males (Einon, 1980; Seymoure, Dou, \& Juraska, 1996; Tees, Midgley, \& Nesbitt, 1981; Williams, Barnett, \& Meck, 1990), and others have not (Juraska, Henderson, \& Muller, 1984; Van Haaren, Wouters, \& Van De Poll, 1987). There are, of course, a number of possible explanations for these inconsistencies, including differences in the apparatus used, details of the task used, and the use of gonadectomized subjects in some, but not other, instances. Although the finding of higher male choice accuracy in spatial tasks is not consistent, all studies that have shown a sex difference have shown it to favor males.

A criticism raised in the use of the RAM to look at sex differences in spatial performance is that any observed

This experiment was completed in partial fulfillment of the requirements for the degree of master of science by B.K.L. under the direction of M.F.B. The authors thank Morgan Terrinoni for his creation of Figure 1 and Inge Ward and Byron Ward for their advice during the development of this experiment. Correspondence should be addressed to M. F. Brown, Department of Psychology, Villanova University, 800 Lancaster Ave., Villanova, PA 19085 (e-mail: mbrown@email.vill.edu). sex difference in the RAM may be due to factors not specifically related to spatial processing. For example, the consistent finding that female rats exhibit higher levels of both activity level and exploratory behavior are possible explanations for the sex differences observed in RAM performance (Beatty, 1992; Wisniewski, 1994).

In addition to the potentially confounding influence of activity level, research into the effects of the extramaze environment on maze performance suggests that the RAM task elicits sex differences in spatial stimulus control that may complicate comparisons of male and female spatial processing. Specifically, Williams et al. (1990) found that male rats are controlled to a greater extent by the geometric features of the room and to a lesser extent by the extramaze landmark cues provided. In their experiments, little disruption of male performance was found when the extramaze cues were removed, as long as the geometric properties of the room remained stable. Female rats, however, could rely on either room geometry or objects in the room (landmarks) to navigate in the maze. It is possible that this difference in the types of spatial cues used by males and females might account, at least in part, for the inconsistencies in the literature on sex differences in the RAM. Specifically, superior performance by males in the RAM may depend on the extent to which an extramaze environment supports the use of geometric cues rather than landmarks.

The pole box maze, introduced by Brown and Terrinoni (1996) and illustrated in Figure 1, appears to isolate an aspect of spatial performance that requires representation of spatial patterns. Thus, it is possible that performance in the pole box may provide insight into sex differences in spatial processing and spatial representation that have not been forthcoming from research employing the RAM. The pole box maze consists of a $5 \times 5$ matrix of vertical poles in a rectangular box, each pole being just tall enough that a rat must rear up on its hind legs to reach the top of the pole. A reward pellet can be placed into a well at the top of the pole so the rat is unable to see the bait in the pole while traveling through the maze. On 




Figure 1. Cutaway illustration of the pole box maze.

each trial, a subset of the 25 poles are baited, and the baited poles always form a particular spatial pattern (e.g., a square formed by four adjacent poles). Aside from this spatial pattern, the identity of the poles baited on each trial is unpredictable. Control of choice behavior by the pattern is measured as the extent to which rats tend to choose poles that are consistent with the pattern. Using simple square and linear patterns, Brown and Terrinoni found that the choices of male rats were controlled by these configurations. Rats were found to choose poles baited according to the geometric rule significantly more often than would be expected by chance alone. Brown and Terrinoni provided several lines of evidence that this tendency could not be accounted for in terms of an ability to perceive the presence of the pellets on top of the poles, and they therefore concluded that it requires some form of representation of the spatial configuration of baited poles. Control by the spatial pattern in which food is found may prove to be an important component of natural foraging processes (see, e.g., Healy \& Hurly, 1998; Kamil \& Jones, 1997).

The precise nature of the spatial representations and corresponding processes involved in the pole box maze remain to be worked out. Representation of the spatial configuration of baited locations in the pole box maze probably has much in common with spatial representation as it has been studied in other behavioral paradigms, but also appears to have important unique properties. For example, most studies of spatial representation involve the spatial relations among landmarks or between landmarks and the goal location (see Thinus-Blanc, 1996, for a review). In the pole box maze, landmarks are explicitly excluded as a possible source of behavioral control. More closely related to the ability exhibited in the pole box maze is the control of choice by the geometric features of the global environment in which spatial choices are made (Cheng, 1986; Williams et al., 1990's application of this idea to sex differences is mentioned above). Like landmarks, control of choice by the global environment requires perception of the cue (landmark or environmental shape). Control by the spatial configuration of the goals in the pole box maze, however, cannot be based on perceived cues. It remains possible, however, that the shape of the pole box maze or the room in which it is located is used by the rat to calibrate its current location with respect to the represented pattern of goal locations.

In the present experiment, we employed the square baiting configuration used by Brown and Terrinoni (1996) to compare spatial search and spatial pattern learning in male and female rats. The efficiency with which rats find the first baited pole is a matter of the efficiency of general spatial search processes. Prior to discovery of the first baited pole, the location of baited poles is unknowable. Once one or more baited poles have been discovered, however, their location provides information about the location of the remaining baited poles. Of particular interest in the present experiment, as in those of Brown and Terrinoni, was whether rats' choices were controlled by this information. If so, such control demonstrates representation of the spatial pattern in which poles are baited. In the present experiment, the performance of male and female rats in this spatial task was compared, with par- 
ticular consideration of whether the choices of male rats were controlled by the spatial pattern to a greater extent than the choices of females.

\section{METHOD}

\section{Subjects}

Fifteen male and 15 female rats, obtained from Harlan SpragueDawley (Indianapolis), were used. The rats were from five litters, with 3 males and 3 females coming from each. All animals were experimentally naive and approximately 3 months of age when the experiment began. Animals were housed in same-sex groups of 3 and maintained on a reverse 12:12-h light:dark cycle with the lights going off at 0700 . Animals were maintained on an ad-lib diet until 2 weeks prior to testing. At that time, the daily diet was reduced so that the animals were maintained at $85 \%-90 \%$ of their free feeding weight as determined by growth curves (Harlan Sprague- Dawley, Inc., 1996).

\section{Apparatus}

The pole box was a rectangular arena measuring $180 \mathrm{~cm} \times$ $76 \mathrm{~cm} \times 33 \mathrm{~cm}$ (tall). The arena was painted flat black and constructed of $1.5-\mathrm{cm}$-thick plywood, with the top of the box open. A thin layer of bedding material covered the floor of the maze. A matrix of vertical poles was located in the center of the apparatus, with each end of the arena left open, allowing room for the rat to be placed at the start of each trial. Each pole in the $5 \times 5$ matrix was made of an unpainted wooden dowel, $15.5-\mathrm{cm}$ tall and $1.2 \mathrm{~cm}$ in diameter. Previous experiments in our laboratory have shown this height to allow the rats to explore the well at the top of each pole with their tongues while rearing up on their hind legs. The well at the top of each pole is deep enough that a reward pellet is out of view of a rat as it traverses the maze. Poles in each row and column are separated by $13 \mathrm{~cm}$. The maze was mounted on a Lazy Susan to allow it to be easily rotated to randomize the orientation of the extramaze environment. The maze was located in the center of a small room with several other objects. Lighting was provided by fluorescent tubes.

During the early phases of the study, a training apparatus identical to the one used by Brown and Terrinoni (1996) was used inside the maze box. The training apparatus consisted of poles identical to those used in their experiment except in height. The poles were attached in series, from shortest to tallest $(2.0,4.5,12.5$, and $15.5 \mathrm{~cm})$. Each of these training poles was separated by $7 \mathrm{~cm}$ and was attached to a plywood base. These poles could be easily placed in the box as a single unit and the base covered with bedding material.

\section{Training Procedure}

For each of 3 days prior to the beginning of the training procedure, the rats were given (in their home cage) $10-20$ of the reward pellets to be used in the experiment (Bio-Serve 45-mg sucrose pellets) to ensure that they would be familiar with the reward. After these initial 3 days, Phase 1 of training began. During this phase, the rats were placed at one randomly chosen end of the box and allowed to explore the experimental apparatus. The four-pole training apparatus was placed in the same end of the box, and each pole of the training apparatus, as well as all 25 poles in the matrix of poles, was baited with a single reward pellet. During the first two training trials, several reward pellets were placed on the floor of the apparatus near the four-pole training apparatus. Each animal participated in two trials per day with $3 \mathrm{~min}$ between trials. The orientation of the maze was randomly selected from among eight equally spaced orientations with respect to the testing room. This was done to minimize and randomize any effects of extramaze cues on the behavior of the test animals.
During both training and testing, the sequence of the sex of the tested rats was counterbalanced in an attempt to balance any possible pheromonal effects on behavior. In addition, after each animal was tested, the bedding material was moved about within the box. Phase 1 of the training period ended when the rat had successfully consumed the pellets on all four poles of the training apparatus and 10 poles within the experimental apparatus within $3 \mathrm{~min}$ during five consecutive trials.

The square configuration of baited poles was introduced during Phase 2 of training. During the 1 st day of this phase, a barrier was placed between the poles so that only two rows were available to the rat. Four spatially adjacent poles were baited in a square configuration. In addition, during this initial day of Phase 2 , while only two rows were available, the training apparatus remained, with each of the four poles baited. From the four possible squares among the 10 poles, one was randomly chosen to be baited. The rat was then placed in the box and allowed to search freely among the available poles. During the 2 nd day of Phase 2, the barrier was moved so that three rows of poles were available to the rat. A square configuration of four adjacent poles was again randomly chosen. On the 4th day of Phase 2, the rat had four rows of poles available, which again had one of the possible squares randomly baited. All trials ended when the rat had discovered all four of the baited poles or $5 \mathrm{~min}$ had elapsed without a choice. The rat completed training and progressed to the testing procedure when it successfully completed three out of four consecutive trials.

\section{Testing Procedure}

Following training, 90 trials of testing were conducted. One randomly chosen set of four poles in the square configuration was baited prior to each trial. The animal was placed onto a randomly chosen side of the testing apparatus and allowed to freely explore the arena. Each trial ended when all four of the baited poles had been chosen. As in Brown and Terrinoni's (1996) experiments, a choice was defined by the rat touching the top of a pole with its snout. The experimenter recorded the sequence of choices made during the trial. All trials were videotaped using a camcorder mounted above the apparatus, so that any ambiguous choice sequences could be resolved by the experimenter (this was needed very rarely).

Each animal was tested for two trials each day with an intertrial interval of approximately $10 \mathrm{~min}$. The order in which rats were tested

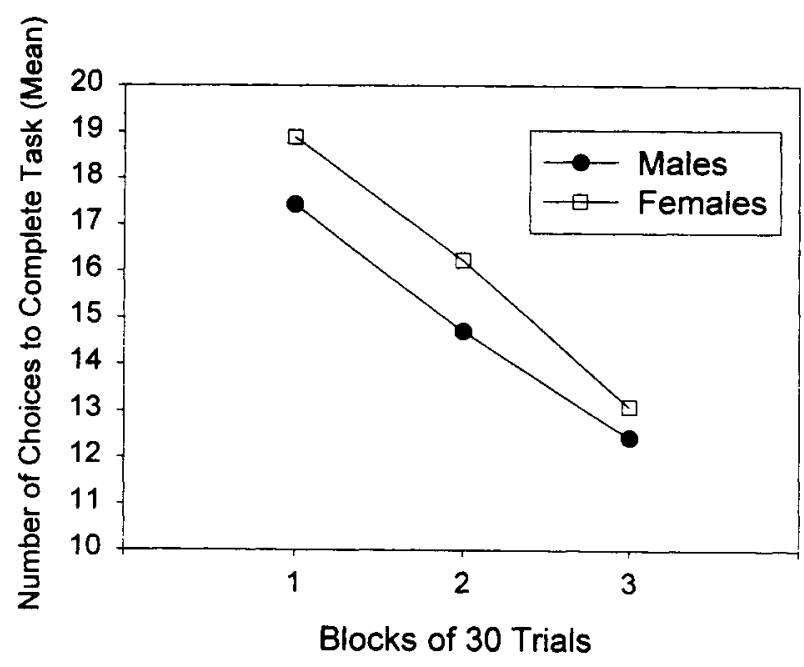

Figure 2. The mean number of choices required to complete the task for male and female rats, plotted over trial blocks. 
was counterbalanced by sex, with all 15 subjects of one sex being tested first, followed by all 15 subjects of the other sex. The order in which the two sexes were tested alternated over days.

\section{RESULTS}

\section{Number of Choices Required to Complete Task}

The most straightforward measure of performance in this task is the number of choices required to complete the task. As shown in Figure 2, an improvement in performance was found over the course of trial blocks $[F(2,56)=$ $79.4, p<.001]$. Although female rats appear to have required more choices than the males to complete the task, there was no reliable difference between males and females in the number of choices to complete the task $[F(1,28)=$ $2.36, p=.13]$. In addition, there was no significant interaction between the effects of these variables $[F(2,56)<1]$. This analysis includes all choices made and therefore does not isolate control by the baiting pattern.

\section{Discovery Transitions}

More precise information about choice processes is provided by analysis of the number of choices that occurred between discoveries of baited poles (discovery transitions). Figure 3 displays the discovery transitions for both sexes across the three trial blocks. During the first transition-that is, from the placement of the rat within the box to discovery of the first baited pole (panel A) female rats were found to make significantly fewer choices than males $[F(1,28)=5.84, p<.05]$. This finding remained constant across all three trial blocks [no block $\times$ sex interaction was found, $F(2,56)=1.11$ ]. In addition, a main effect of block was found, with rats requiring fewer choices to discover the first baited pole across trial blocks $[F(2,56)=8.93, p<.001]$.

The second discovery transition consists of choices made by the rats following discovery of the first baited pole and concluding with the discovery of the second baited pole (Figure 3, panel B). No sex difference in the number of choices to discover the second baited pole was found $[F(1,28)=1.29]$. Again, a main effect of block was found, with fewer choices required to locate the second baited pole as the trial blocks progressed $[F(2,56)=$ $20.24, p<.001]$. A block $\times$ sex interaction was not present $[F(2,56)<1]$.

The third discovery transition consists of choices following discovery of the second baited pole and concluding with the discovery of the third baited pole (Figure 3, panel C). It is the choices that the rats made following discovery of the second baited pole and following the discovery of the third baited pole that are of primary interest in this study. Data from these two choice transitions allow control of choices by the baiting pattern to be isolated, using the measures described in the next section. A sex $\times$ block analysis of variance (ANOVA) revealed that male rats required significantly fewer choices than females before discovery of the third baited pole $[F(1,28)=6.05, p<.05]$. In addition, across trial blocks

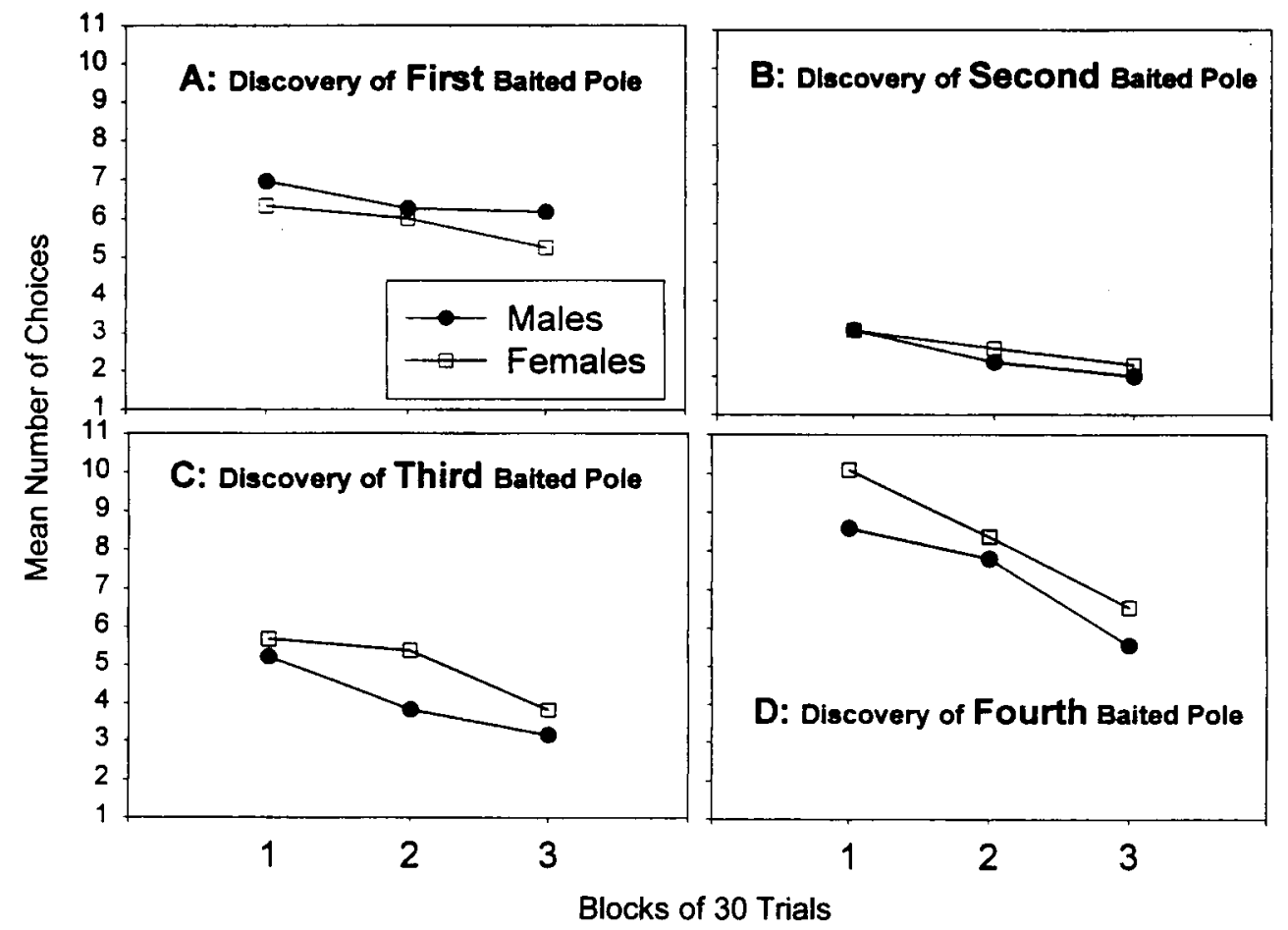

Figure 3. The mean number of choices leading up to and including discovery of the first (panel $A$ ), second (panel B), third (panel C), and fourth (panel D) baited poles. Data are shown for male and female rats over the course of trial blocks. 
the number of choices required to discover the third baited pole decreased significantly $[F(2,56)=28.56, p<.001]$. No block $\times$ sex interaction was found $[F(2,56)=2.50$, $p=.09]$.

The sex $\times$ block ANOVA for the fourth discovery transition (Figure 3, panel D) did not reveal a significant sex difference in the number of choices required to discover the fourth baited pole $[F(2,56)=2.31, p=.14]$. There was an improvement over trial blocks $[F(2,56)=19.94$, $p<.001]$. As with the other three discovery transitions, no block $\times$ sex interaction was found $[F(2,56)<1]$.

\section{Control by Square Pattern}

The central analysis of this study explored the ability of the rats to make choices according to the rules of the baiting pattern. Figure 4 displays the logic of this analysis. After the discovery of the second baited pole, the two poles are either aligned in a row or column (panel A) or make

\section{A: Two Baited Poles Discovered (aligned)}



\section{B: Two Baited Poles Discovered (corners)}

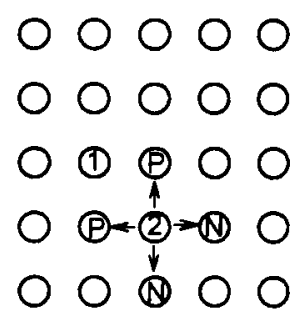

\section{C: Three Baited Poles Discovered}

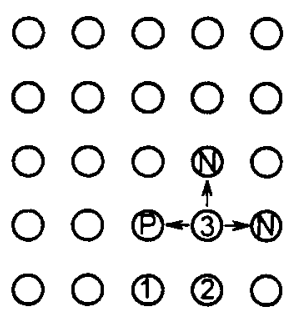

Figure 4. Schematic illustration of poles and moves that were used in the primary analysis. The numbers indicate the order in which the baited poles were discovered. $P$, possibly baited pole; $N$, not possibly baited pole. As indicated by the arrows, only poles adjacent to the just-discovered pole were used in the analysis. up the opposite corners of the baited square (panel B). In both situations, there are two poles adjacent to the second discovered baited pole (identified as 2 in Figure 4) that could possibly be baited according to the rule. There are also either one (panel A) or two (panel B) poles that cannot possibly be baited according to the rule. Once the third baited pole is discovered, there is a single possibly baited pole and two adjacent poles that cannot possibly be baited (panel C). Analysis of the differences between the expected and obtained proportions allows measurement of control of choices by the spatial pattern. The two proportions were constructed using the methods employed by Brown and Terrinoni (1996). The analysis did not include choices made to diagonal poles because choices to diagonal poles occurred rarely and because inclusion of them would have greatly complicated the analysis. Data from moves immediately following the discovery of the second baited pole were analyzed separately from moves directly following the discovery of the third baited pole. Choices immediately following discovery of the second and third baited poles were the only choices used in the analysis.

The obtained proportion is a measure of the tendency of the rat to choose poles that could possibly be baited according to the geometric rule. Only choices of poles immediately following discovery of the second or third baited poles are considered in calculating this proportion. In addition, the measure considers only moves from the discovered baited pole to previously unvisited, adjacent poles. The proportion of moves conforming to these restrictions made to poles that conformed to the square pattern was calculated for each rat during each trial block. It is important to note that not all poles conforming to the square pattern were actually baited. For example, when two adjacent baited poles were discovered, there were two possibly baited poles adjacent to the baited poles just discovered, but only one of these two poles was actually baited.

These obtained proportions were compared with corresponding expected proportions, which were an estimate of the proportions expected on the basis of chance. The expected proportions were determined by considering the previously unvisited, adjacent poles available at the time that moves included in the obtained proportions were made. Specifically, for each rat during each trial block, the number of previously unvisited, possibly baited, adjacent poles was divided by the total number of previously unvisited adjacent poles. For example, the combination of possible choices illustrated in panels $A$ and $B$ of Figure 4 would lead to the expected proportion of $4 / 7=.57$.

Figure 5 displays the mean expected and obtained proportions following discovery of the second baited pole. A proportion $\times$ sex $\times$ block ANOVA revealed no triple interaction $[F(2,56)<1]$. Critically, a proportion $\times$ sex interaction was found, indicating that the difference between the expected and obtained performance was larger for males than for females $[F(1,28)=21.18, p<.001]$. In order to examine performance by each sex separately, 
two proportion $\times$ block ANOVAs were employed. A significant interaction was found between proportion and block for males, indicating increased control by the baiting pattern across blocks $[F(2,28)=3.38, p<.05]$. This interaction was not present for the females $[F(2,28)=$ $3.00, p=.066]$. There was, however, an effect of proportion for the females $[F(1,28)=31.96, p<.001]$, indicating that their choices were controlled by the baiting pattern.

Figure 6 displays the proportions expected and obtained following discovery of the third baited pole. As was the case following discovery of the second baited pole, a proportion $\times$ sex $\times$ block ANOVA revealed no triple interaction $[F(2,56)<1]$. Subjects again were found to choose poles conforming to the pattern more often than would be expected by chance, as indicated by a main effect of proportion $[F(1,28)=95.71, p<.001]$. However, the lack of a proportion $\times$ sex interaction confirms the impression given by Figure 6 that there was no evidence of a sex difference $[F(1,28)=1.31, p=.26]$. The tendency to choose poles conforming to the pattern increased as the trial blocks progressed, as evidenced by a block $\times$ proportion interaction $[F(2,56)=5.23, p<.01]$.

\section{Discovery of the First Baited Pole}

Several analyses were conducted in an attempt to better understand the fact that females located the first baited pole with fewer choices than did males. This finding indicates that, when no information about the location of baited poles is available, females search more efficiently than males. Figure 7 shows the results of three analyses conducted to examine possible explanations for this difference in search efficiency. All three of these analyses were restricted to choice behavior prior to discovery of the first baited pole. The first looked at a measure of the tendency of rats to avoid revisits to poles cho-

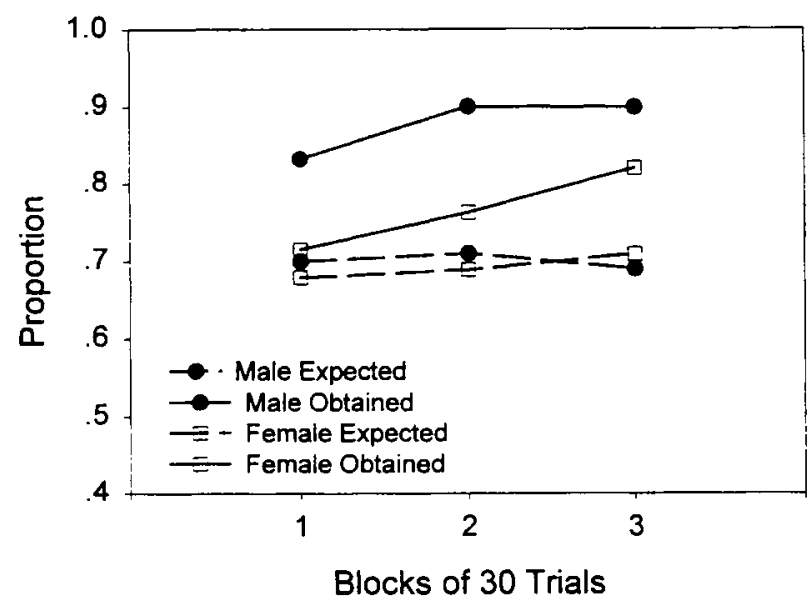

Figure 5. Expected and obtained proportions of choices immediately following discovery of the second baited pole that were directed to poles conforming to the square pattern. Only moves to adjacent, previously unvisited poles are included in the analysis (see text for details).

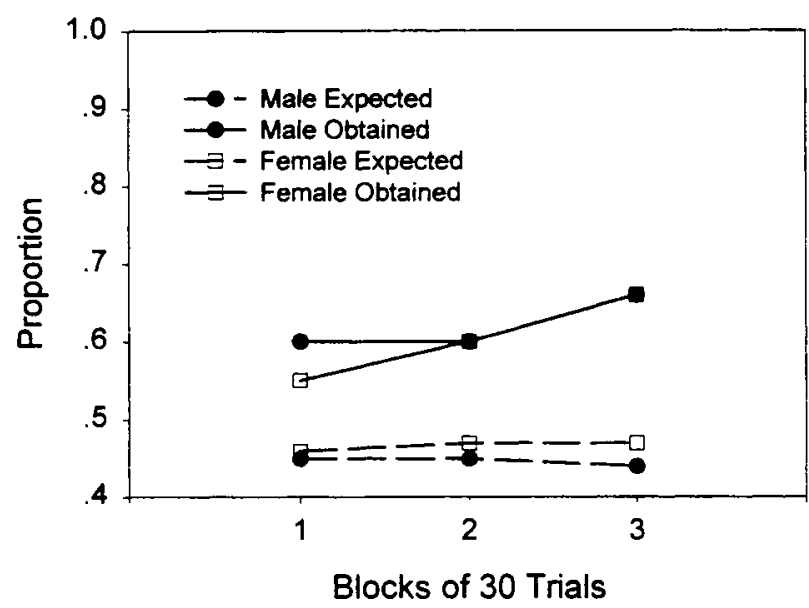

Figure 6. Expected and obtained proportions of choices immediately following discovery of the third baited pole that were directed to poles conforming to the square pattern. Only moves to adjacent, previously unvisited poles are included in the analysis (see text for details).

sen earlier (Figure 7, panel A). This is analogous to the well-studied ability of rats to avoid revisits to maze arms in the radial arm maze. There was an improvement in the ability to avoid revisits to poles over the course of trial blocks $[F(2,56)=2.79, p<.05]$, but no evidence of a difference between males and females $[F(1,28)=1.25]$, nor of an interaction between the effects of these variables $[F(2,56)<1]$.

The second analysis examined the proportion of choices leading up to discovery of the first baited poles that were to adjacent poles (Figure 7, panel B). There was an increase in the tendency to choose adjacent poles over the course of trial blocks $[F(2,56)=46.92, p<.001]$. The tendency was greater for male rats $[F(1,28)=5.13, p<$ $.05]$. There was no interaction between the effects of these variables $[F(2,56)<1]$.

The tendency to choose poles on the edge of the matrix ( 16 of the 25 poles were on the edge) was also examined in an attempt to determine whether there were differences in thigmotaxis that might have contributed to the sex difference in spatial search (Figure 7, panel C). There was a decrease in the proportion of choices that were to poles on the edge of the matrix over the course of trial blocks $[F(2,56)=43.36, p<.001]$. The tendency to choose edge poles was not significantly different for male and female rats $[F(1,28)=4.19]$. There was no interaction between the effects of these variables $[F(2,56)<1]$.

\section{DISCUSSION}

The results of this study indicate that both males and females can be controlled by a representation of the spatial pattern in which hidden food items have been previously found. That is, both male and female rats chose poles that corresponded with the baiting pattern more 

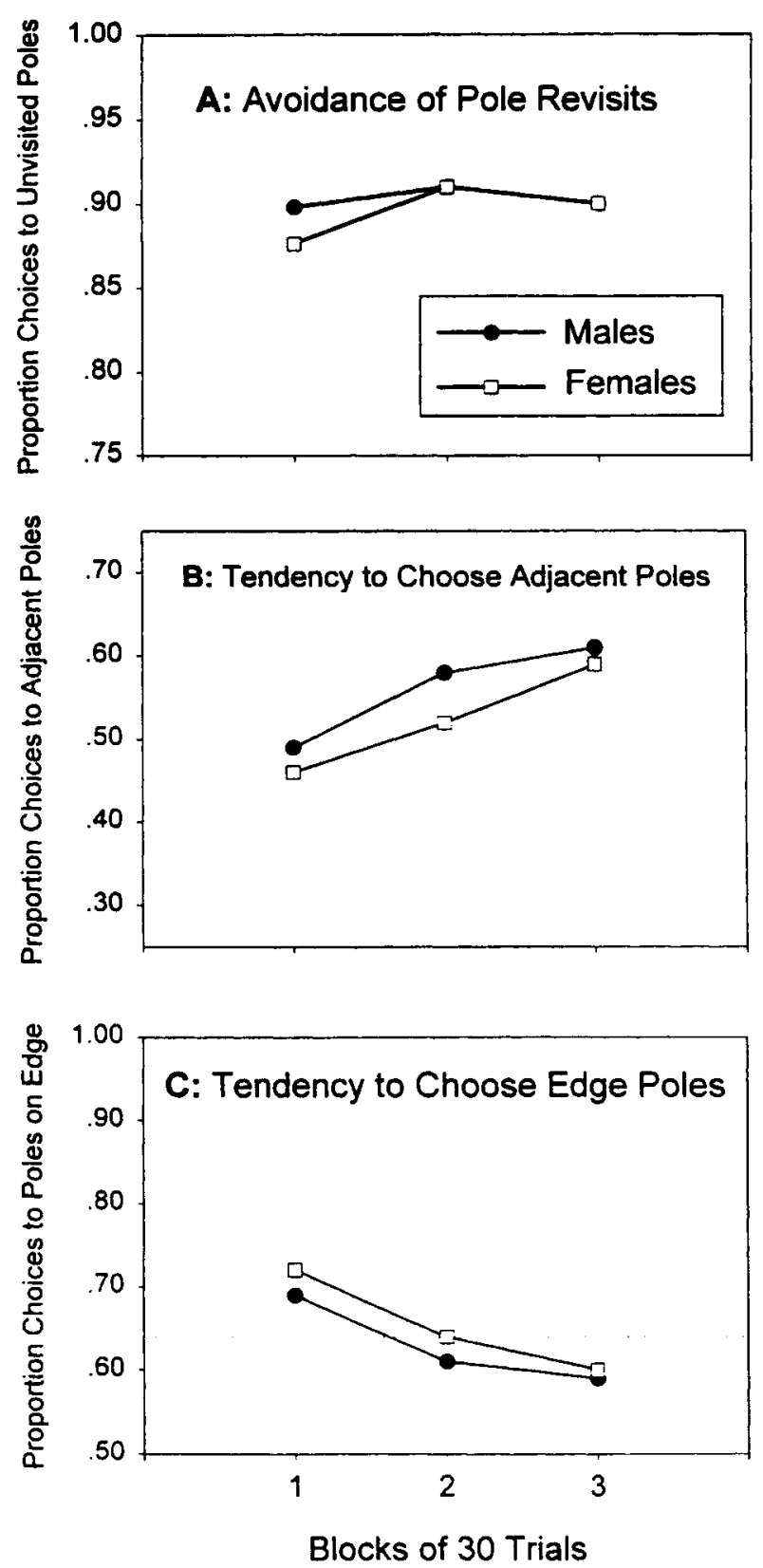

Figure 7. Analyses of selected aspects of the search process leading up to discovery of the first baited pole. Measures of the tendencies to avoid revisits to poles (panel $\mathrm{A}$ ), choose poles adjacent to the previous choice (panel B), and choose poles on the edge of the pole matrix (panel $C$ ) are shown for male and female rats over trial blocks. The reference line in panel $\mathrm{C}$ shows the value expected on the basis of chance.

often than would be expected by chance. In addition, a sex difference was found, with males being better able to utilize the baiting pattern to guide behavior following discovery of the second baited pole. This sex difference appeared rapidly: It was apparent within the first block of 30 trials. The magnitude of this difference remained consistent throughout the remainder of the experiment, with both sexes becoming better able to use the baiting pattern as the trials progressed.

Interestingly, without the possibility of control by the spatial pattern (i.e., during the search for the first baited pole), female rats were found to discover the first baited pole more efficiently than male rats. The fact that males and females exhibited relative superiority on different aspects of the experimental task probably accounts for the lack of a sex difference in the overall measure of choice accuracy (number of choices required to complete the task). Control of choices by the spatial pattern clearly cannot facilitate discovery of the first baited pole, so female superiority on this part of the task must be due to some other mechanism. At least part of this difference might be due to the stronger tendency of male rats to choose adjacent poles. Given that baited poles are adjacent to other baited poles, there is an advantage to searching poles not adjacent to a pole already determined not to be baited. Whether females are more sensitive to this contingency or female search tendencies just happen to be more compatible with this detail of the pole box task is unknown. One possibility is that this tendency is related to the higher levels of exploratory behavior found in female rats: Higher levels of exploratory behavior might be expected to produce fewer moves to adjacent poles.

Discovery of the third and fourth baited poles are most directly diagnostic of control by the square spatial pattern in which poles were baited, and it was within the confines of these two choice transitions that the sex difference was hypothesized to be found. As would be predicted by the view that male spatial ability is superior to that of females, male rats were better able to locate a third baited pole after finding two of them. However, there was no evidence for a sex difference in the ability to locate the last baited pole after finding the third one. A priori, location of both of these poles could be facilitated by knowledge of the baiting pattern. It is unclear why the sex difference did not continue throughout the task. However, one possibility is that females were able to compensate for the sex difference found following discovery of the second baited pole by some other mechanism. Following discovery of the second baited pole, the rat has access to relatively little information about the location of the square pattern (i.e., the two discovered poles and perhaps information acquired when surrounding poles are found not to be baited). During location of the fourth baited pole, more information is present. At that point there are at least three spatial cues (the three discovered poles, and perhaps additional "misses" to unbaited poles) to help guide behavior. It may be that this additional information is critical in allowing female performance to match that of males.

The results of this study are consistent with the general view that male rats perform more accurately than female rats on tasks that require spatially organized representations. However, as described in the introduction, it is not yet clear how the cognitive processes measured in the present study are related to those involved in other 
spatial tasks, such as those that have been studied in the RAM. Most research suggests that performance in the RAM is based on the use of extramaze cues as landmarks to help guide behavior (e.g., Brown, 1992; Suzuki, Augerinos, \& Black, 1980; Williams et al., 1990). Whether these cues are represented in a spatially organized fashion, however, is a matter of debate (Brown, 1992). Furthermore, a variety of other spatial and nonspatial mechanisms are involved in RAM performance, depending on details of the experimental conditions (Brown \& Bing, 1997; Brown \& Moore, 1997). The results of an experiment by Dallal and Meck (1990) suggest that rats may represent spatial patterns in the RAM in a manner that is at least similar to the spatial representations implied by the present results. In Dallal and Meck's experiment, rats learned to avoid visits to maze arms that had never been baited. In a transfer test, rats performed more accurately in a novel extramaze environment when the never-baited arms were arranged in the same spatial configuration during the transfer test as they had been during training. This suggests that the spatial pattern of never-baited arms was represented, just as the spatial pattern of baited poles is represented in the pole box.

Additional research will be required to determine the precise nature of the mechanisms allowing rats to choose poles consistent with spatial patterns in the pole box, as well as to determine which aspects of these mechanisms account for the sex differences in performance reported here. However, the present results indicate that the pole box is a valuable tool for the exploration of sex differences in spatial ability and spatial representation. Previous sex difference research in rodents can be criticized for its lack of consideration of various exploratory and other nonspatial performance factors that may mask or interfere with the ability to measure sex differences in processes that are specifically spatial. The pole box task and the analytic procedures used in the present work allow control of choice by spatial patterns to be isolated from other factors affecting performance, such as the search behavior that led to female superiority in discovery of the first baited pole.

\section{REFERENCES}

BEATTY, W. W. (1979). Gonadal hormones and sex differences in nonreproductive behaviors in rodents: Organizational and activational influences. Hormones \& Behavior, 12, 112-163.

Beatty, W. W. (1984). Sexual dimorphism in spatial behavior. In G. J. de Vries, J. P. C. de Bruin, H. B. M. Uylings, \& M. A. Corner (Eds.), Sex differences in the brain. The relationship between structure and function (Progress in Brain Research, Vol. 61, pp. 320-329). Amsterdam: Elsevier.

BEATtY, W. W. (1992). Gonadal hormones and sex differences in nonreproductive behaviors. In A. A. Gerall, H. Moltz, \& I. L. Ward
(Eds.), Sexual differentiation (Handbook of Behavioral Neurobiology, Vol. 11 , pp. 85-128). New York: Plenum.

Brown, M. F. (1992). Does a cognitive map guide choices in the radialarm maze? Journal of Experimental Psychology: Animal Behavior Processes, 18, 56-66.

Brown, M. F., \& BING, M. N. (1997). In the dark: Spatial choice when access to spatial cues is restricted. Animal Learning \& Behavior, 25 , 21-30.

Brown, M. F., \& Moore, J. A. (1997). In the dark II: Spatial choice when access to extrinsic cues is eliminated. Animal Learning \& Behavior, 25, 335-346.

Brown, M. F., \& Terrinoni, M. (1996). Control of choice by the spatial configuration of goals. Journal of Experimental Psychology: Animal Behavior Processes, 22, 438-446.

CHENG, K. (1986). A purely geometric module in the rat's spatial representation. Cognition, 23, 149-178.

DallaL, N. L., \& MECK, W. H. (1990). Hierarchical structures: Chunking by food type facilitates spatial memory. Journal of Experimental Psychology: Animal Behavior Processes, 16, 69-84.

EINON, D. (1980). Spatial memory and response strategies in rats: Age, sex and rearing differences in performance. Quarterly Journal of Experimental Psychology, 32, 473-489.

HALPERN, D. F. (1992). Sex differences in cognitive abilities. Hillsdale, NJ: Erlbaum.

Harlan Sprague-Dawley, Inc. (1996). Product guide [Brochure]. Indianapolis, IN: Author.

Healy, S. D., \& Hurly, T. A. (1998). Rufous hummingbirds' (Selasphorus rufus) memory for flowers: Patterns or actual spatial locations? Journal of Experimental Psychology: Animal Behavior Processes, 24, 396-404.

Juraska, J. M., Henderson, C., \& Muller, J. (1984). Differential rearing experience, gender, and radial maze performance. Developmental Psychobiology, 17, 209-215.

KamiL, A. C., \& JonEs, J. J. (1997). The seed-storing Corvid Clark's nutcrackers learns geometric relationships among landmarks. $\mathrm{Na}$ ture, 390, 276-279.

Seymoure, P., Dou, H., \& Juraska, J. M. (1996). Sex differences in radial maze performance: Influence of rearing environment and room cues. Psychobiology, 24, 33-37.

Suzuki, S., Augrerinos, G., \& Black, A. H. (1980). Stimulus control of spatial behavior on the eight-arm maze in rats. Learning \& Motivation, 11, 1-18.

TeEs, R. C., Midgley, G., \& Nesbitt, J. C. (1981). The effects of early visual experience on spatial maze learning in rats. Developmental Psychobiology, 14, 425-438.

Thinus-Blanc, C. (1996). Animal spatial cognition: Behavioural and brain approaches. Singapore: World Scientific.

Van Haaren, F., Wouters, M., \& Van De Poll, N. E. (1987). Absence of behavioral differences between male and female rats in different radial-maze procedures. Physiology \& Behavior, 39, 409-412.

Williams, C. L., BarnetT, A. M., \& MECK, W. H. (1990). Organizational effects of early gonadal secretions on sexual differentiation in spatial memory. Behavioral Neuroscience, 104, 84-97.

WiLliams, C. L., \& MECK, W. H. (1991). The organizational effects of gonadal steroids on sexually dimorphic spatial ability. Psychoneurendocrinology, 16, 155-176.

WISNIEWSKI, C. (1994). Gender and criterion differences in the radialarm maze. Unpublished master's thesis, Villanova University, Villanova, PA.

(Manuscript received August 6, 1998; revision accepted for publication March 19, 1999.) 